\title{
Increased leukocyte rho kinase (ROCK) activity and endothelial dysfunction in cigarette smokers
}

\author{
Takayuki Hidaka $^{1}$, Takaki Hata ${ }^{1}$, Junko Soga ${ }^{1}$, Yuichi Fujii ${ }^{1}$, Naomi Idei ${ }^{1}$, Noritaka Fujimura ${ }^{1}$, Yasuki Kihara ${ }^{1}$, \\ Kensuke Noma ${ }^{2}$, James K Liao $^{3}$ and Yukihito Higashi ${ }^{2}$
}

Rho-associated kinases (ROCKs) have been shown to be involved in the pathogenesis of atherosclerosis. Although smoking is associated with endothelial dysfunction and ROCK inhibitors improve endothelial function in smokers, it is not known whether ROCK activity is increased in smokers and whether this correlates with endothelial dysfunction. The purpose of this study was to evaluate the relationship between ROCK activity and endothelial function in smokers. We evaluated flow-mediated vasodilatation (FMD) using ultrasonography and ROCK activity in peripheral leukocytes using western blot analysis in 14 male smokers (28.1 \pm 3.9 years) and 15 healthy male non-smokers $(28.3 \pm 3.6$ years). ROCK activity was defined as the ratio of phosphomyosin-binding subunit (MBS) on myosin light-chain phosphatase to total MBS. FMD was significantly less in smokers than in non-smokers ( $4.7 \pm 3.1$ vs. $9.0 \pm 3.8 \%, P=0.005)$. Nitroglycerine-induced vasodilation was similar in the two groups. ROCK activity was greater in smokers than in non-smokers $(0.78 \pm 0.27$ vs. $0.54 \pm 018, P=0.012)$. The expression of total MBS, ROCK 1 and ROCK2 were similar in the two groups. ROCK activity correlated with systolic blood pressure $(r=0.42, P=0.039)$. Multiple regression analysis revealed that smoking is an independent predictor of ROCK activity. There was a significant correlation between FMD and ROCK activity $(r=-0.42, P=0.035)$. No other variable was correlated with FMD. These findings suggest that ROCK activity is enhanced by smoking and is a predictor of endothelial function.

Hypertension Research (2010) 33, 354-359; doi:10.1038/hr.2010.3; published online 5 February 2010

Keywords: endothelial function; Rho-associated kinase; smoker

\section{INTRODUCTION}

Cigarette smoking is a major risk factor in the development of atherosclerosis ${ }^{1,2}$ and independently increases clinical cardiovascular morbidity and mortality. ${ }^{3}$ Several lines of evidence have shown that cigarette smoking alters hemostasis, causes inflammation and oxidative stress in vascular walls ${ }^{4}$ and impairs endothelial function., In addition, some studies have shown that cigarette smoking increases the numbers of circulating leukocytes and inflammatory markers such as C-reactive protein (CRP), interleukin-6 and soluble intercellular adhesion molecule type $1.6,7$

Small guanosine triphosphate-binding protein RhoA (ras homolog gene family, member A) mediates various cellular physiologic functions such as cell proliferation, migration, adhesion, apoptosis and contraction, ${ }^{8-11}$ all of which may be involved in the pathogenesis of atherosclerosis. Rho-associated kinases (ROCKs), which consist of two isoforms, ROCK1 and ROCK2, were found to be the immediate downstream targets of RhoA, ${ }^{12}$ The RhoA/ROCK pathway has been shown to be involved in atherosclerotic lesion formation, ${ }^{13}$ vasoconstriction $^{9,14}$ and myocardial hypertrophy ${ }^{15}$ and to be activated in patients with hypertension ${ }^{16}$ and coronary artery disease. ${ }^{11,17}$ Previous studies using ROCK inhibitors such as fasudil or Y-27632 have suggested that ROCK may have an important role in the pathogenesis of cardiovascular disease. ${ }^{9,13,15}$

The vascular endothelium is involved in the release of various vasodilators, including nitric oxide (NO), prostaglandins and endothelium-derived hyperpolarizing factor as well as vasoconstrictors. ${ }^{18} \mathrm{NO}$ has an important role in the regulation of vascular tone, inhibition of platelet aggregation and suppression of smooth muscle cell proliferation. ${ }^{19}$ Endothelial dysfunction is the initial step in the pathogenesis of atherosclerosis, resulting in cardiovascular and cerebrovascular outcomes. Previous studies have shown that activation of the RhoA/ROCK pathway impaired NO bioavailability through inhibition of endothelial NO synthase (eNOS) mRNA stability and eNOS protein phosphorylation at Ser 1177 through the Akt/ phosphoinositide 3-kinase pathway. ${ }^{20}$

Recently, it has been reported that ROCK activity is increased in subjects with metabolic syndrome and in patients with coronary artery disease. ${ }^{11,21}$ In those studies, ROCK activity in peripheral leukocytes was directly assayed by western blot analysis using a specific antibody to phospho-myosin-binding subunit (MBS) on

${ }^{1}$ Department of Cardiovascular Medicine, Hiroshima University Graduate School of Biomedical Sciences, Hiroshima, Japan; ${ }^{2}$ Department of Cardiovascular Physiology and Medicine, Hiroshima University Graduate School of Biomedical Sciences, Hiroshima, Japan and ${ }^{3}$ Vascular Medicine Research Unit, Brigham and Women's Hospital and Harvard Medical School, Boston, MA, USA

Correspondence: Dr Y Higashi, Department of Cardiovascular Physiology and Medicine, Graduate School of Biomedical Sciences, Hiroshima University, 1-2-3 Kasumi, Mimamiku, Hiroshima 734-8551, Japan.

E-mail: yhigashi@hiroshima-u.ac.jp

Received 15 October 2009; revised 27 November 2009; accepted 20 December 2009; published online 5 February 2010 
myosin light-chain phosphatase, which is a downstream target of ROCK. As assessment of ROCK activity in peripheral leukocytes is minimally invasive and does not require pharmacologic intervention using intra-arterial infusion of ROCK inhibitors, it may be useful for evaluating ROCK activity in a clinical setting. Indeed, leukocyte ROCK activity has been shown to correlate inversely with endothelial function. ${ }^{11}$

We previously reported that intra-arterial infusion of the ROCK inhibitor, fasudil, improves endothelial function in smokers. ${ }^{22}$ However, it is not known from these studies whether ROCK activity was higher in smokers and whether it correlated with endothelial dysfunction. The purpose of this study, therefore, is to determine whether ROCK activity is increased in smokers and whether this correlates with endothelial function.

\section{METHODS}

\section{Subjects}

We studied 14 male smokers (mean age: $28.1 \pm 3.9$ years) and 15 healthy male non-smokers (mean age: $28.3 \pm 3.6$ years) who had no history of cardiovascular disease, hypertension or other diseases. Normal blood pressure was defined as systolic blood pressure of $<130 \mathrm{mmHg}$ and diastolic blood pressure of $<80 \mathrm{~mm} \mathrm{Hg}$. The results of physical and routine laboratory examinations of the subjects were normal. None of the subjects were taking oral antioxidant vitamins or vasoactive drugs. Current smokers were defined as smokers who had smoked at least 1 pack-year, with 1 pack-year being defined as 20 cigarettes per day for 1 year. All of the smokers (19.1 \pm 9.2 pack-years) had a current smoking history of more than 5 years and abstained from smoking for at least $3 \mathrm{~h}$ before the measurement of vascular function. We defined non-smokers as subjects who had never smoked. The study protocol was approved by the ethics committee of Hiroshima University Graduate School of Biomedical Sciences. Informed consent for participation in the study was obtained from all subjects.

\section{Measurement of FMD}

All studies were performed in the morning, after overnight fasting, in a quiet, dark and air-conditioned room (constant temperature of $22-25^{\circ} \mathrm{C}$ ). The subjects remained supine throughout the study.

The vascular response to reactive hyperemia in the brachial artery was assessed for ultrasound assessment of endothelium-dependent flow-mediated vasodilatation (FMD). A high-resolution linear artery transducer (13 MHz) was coupled to computer-assisted analysis software (e-TRACKING system, Aloka, Tokyo, Japan) that used an automated edge detection system for measurement of brachial artery diameter. The subjects rested and the right arm was fixed by a special arm-holding device (MIST-100, Saraya, Osaka, Japan). A blood pressure cuff was placed around the forearm. The brachial artery was scanned longitudinally $5-10 \mathrm{~cm}$ above the elbow. When the clearest B-mode image of the anterior and posterior intimal interfaces between the lumen and vessel wall was obtained, the transducer was held at the same point throughout the scan by a special probe holder (MP-PH0001, ALOKA) to ensure consistency of the image. Depth and gain setting were set to optimize the images of the arterial lumen wall interface. When the tracking gate was placed on the intima, the artery diameter was automatically tracked and the waveform of diameter changes over the cardiac cycle was showed in real time using the FMD mode of the e-TRACKING system. This allowed the ultrasound images to be optimized at the start of the scan and the transducer position to be adjusted immediately for optimal tracking performance throughout the scan. The software could accommodate angulations of the artery from -20 to $+20^{\circ}$ relative to the perpendicular. Pulsed Doppler flow was assessed at baseline and during peak hyperemic flow, which was confirmed to occur within $15 \mathrm{~s}$ after cuff deflation. The Doppler flow signals were captured with customized equipment. Blood flow velocity was calculated from the color Doppler data and was showed as a waveform in real time. The baseline longitudinal image of the artery was acquired for $30 \mathrm{~s}$ and then the blood pressure cuff was inflated to $50 \mathrm{~mm} \mathrm{Hg}$ above systolic pressure for $5 \mathrm{~min}$. The longitudinal image of the artery was recorded continuously until $5 \mathrm{~min}$ after cuff deflation. Pulsed Doppler velocity signals were obtained for $20 \mathrm{~s}$ at baseline and for $10 \mathrm{~s}$ immediately after cuff deflation. Changes in brachial artery diameter were immediately expressed as percent change relative to the vessel diameter before cuff inflation. FMD was automatically calculated as the percent change in peak vessel diameter from the baseline value. \%FMD (peak diameter-baseline diameter/baseline diameter) was used for analysis. Blood flow volume was calculated by multiplying the Doppler flow velocity (corrected for the angle) by heart rate and vessel cross-sectional area $\left(-r^{2}\right)$. Reactive hyperemia was calculated as the maximum percentage increase in flow after cuff deflation compared with baseline flow. After a 10-min period to allow baseline conditions of the brachial artery to be reestablished, another baseline scan was performed. The response to nitroglycerine (NTG) was used for assessment of endothelium-independent vasodilation. After acquiring baseline resting images for $30 \mathrm{~s}$, a sublingual tablet (NTG $75 \mu \mathrm{g}$ ) was given, and images of the artery were recorded continuously for $5 \mathrm{~min}$. The response of the brachial artery diameter to NTG was immediately expressed as percent change relative to vessel diameter. NTG-induced vasodilation was automatically calculated as the percent change in peak vessel diameter from the baseline value. \%NTG (peak diameter-baseline diameter/baseline diameter) was used for analysis. The intra-observer coefficient of variation for the baseline diameter was 3.5\% in the supine position and was $2.7 \%$ in the seated position. The respective interobserver coefficients were 2.6 and $1.9 \%$.

Baseline fasting serum concentrations of total cholesterol, highdensity lipoprotein cholesterol, low-density lipoprotein cholesterol, triglycerides, glucose, insulin, electrolytes, high-sensitivity CRP and 8-hydroxy-2'deoxyguanosine (8-OHdG) were obtained after a 30 -min rest period before the study.

\section{Measurement of ROCK activity}

ROCK activity was assayed in peripheral blood leukocytes as the amount of phospho-Thr853 in the MBS of myosin light-chain phosphatase. Blood was collected at room temperature in heparinized tubes $\left(20 \mathrm{U} \mathrm{ml}^{-1}\right)$ with $10 \mathrm{mmoll}^{-1}$ fasudil (Asahi Chemical Industry, Tokyo, Japan ). Fasudil was added to inhibit ROCK activity and hence further formation of phospho-Thr853 MBS ex vivo. After adding an equal volume of $2 \%$ dextran, the sample was kept at room temperature for $30 \mathrm{~min}$. The supernatant was spun at 1450 r.p.m. for $10 \mathrm{~min}$. Red blood cells in the resulting cell pellet were lysed with the addition of water and spun at 1450 r.p.m. for $10 \mathrm{~min}$ after the addition of Hank's balanced salt solution (Hyclone, Logan, UT, USA). The resulting leukocyte pellet was resuspended in medium 199 (Sigma Chemical, Perth, WA, USA) and counted using a hematocytometer. Cells were fixed in 10\% trichloroacetic acid and $10 \mathrm{mmoll}^{-1}$ dichlorodiphenyltrichloroethane. After centrifugation, the cell pellets were stored at $-80^{\circ} \mathrm{C}$ for western blot analysis. Cells pellets were dissolved in $10 \mu \mathrm{l}$ of $1 \mathrm{moll}^{-1}$ Tris base and then mixed with $100 \mu \mathrm{l}$ of extraction buffer $\left(8 \mathrm{moll}^{-1}\right.$ urea, $2 \%$ sodium dodecyl sulfate, $5 \%$ sucrose and $5 \% 2$ mercaptoethanol). Equal amounts of cell extracts were subjected to $7.5 \%$ sodium dodecyl sulfate-polyacrylamide gel electrophoresis and transferred to nitrocellulose membranes. NIH $3 \mathrm{~T} 3$ cell lysates were used as a positive control and to standardize the results of western blot analyses from several membranes. After serum starvation for $20 \mathrm{~h}$, confluent cells were stimulated with $10 \mu \mathrm{moll}^{-1}$ lysophosphatidic acid for $10 \mathrm{~min}$ and then subsequently fixed and harvested in $10 \%$ trichloroacetic acid and $10 \mathrm{mmoll}^{-1}$ dichlorodiphenyltrichloroethane. After centrifugation at 1450 r.p.m. for $10 \mathrm{~min}$ at $4^{\circ} \mathrm{C}$, precipitates were dissolved in $10 \mu \mathrm{l}$ of $1 \mathrm{moll}^{-1}$ Tris base and mixed with $100 \mu \mathrm{l}$ of extraction buffer. An equal volume of positive control cell lysate was used for each gel. Membranes were incubated with rabbit anti-phospho-specific Thr853-MBS polyclonal antibody (Biosource Invitrogen, Carlsbad, CA, USA) or rabbit anti-MBS polyclonal antibody (Covance Laboratories, Evansville, IN, USA), anti-ROCK2 monoclonal antibody, anti-ROCK1 monoclonal antibody (BD Biosciences, San Jose, CA, USA) or antiactin monoclonal antibody (Sigma). Bands were visualized using the enhanced chemiluminescence system (Amersham-Pharmacia, Buckinghamshire, UK). Images were captured using Adobe Photoshop (Adobe Systems, Inc., San Jose, CA, USA), and the band intensities were quantified using National Institutes of Health Image 1.61 (Bethesda, MD, USA). ROCK activity was expressed as the ratio of phospho-Thr853-MBS in each sample to phospho-Thr853-MBS in each positive control divided by MBS in each sample per MBS in each positive control. 


\section{Analytical methods}

Samples of venous blood were placed in tubes containing sodium EDTA $\left(1 \mathrm{mg} \mathrm{ml}^{-1}\right)$ and in polystyrene tubes. The EDTA-containing tubes were chilled promptly in an ice bath. Plasma was immediately separated by centrifugation at $3100 \mathrm{~g}$ for $10 \mathrm{~min}$ at $4{ }^{\circ} \mathrm{C}$, and serum was separated by centrifugation at $1000 \mathrm{~g}$ for $10 \mathrm{~min}$ at room temperature. Samples were stored at $-80^{\circ} \mathrm{C}$ until the time of assay. Serum concentrations of total cholesterol, triglycerides, high-density lipoprotein cholesterol, low-density lipoprotein cholesterol, glucose and electrolytes were determined by routine chemical methods. Serum concentration of high-sensitivity CRP was measured by a high sensitive nephelometry assay using a CRP kit (Dade Behring, Deerfield, IL, USA). Serum concentration of 8OHdG was also assayed by ELISA using 8-OHdG kits (Nihon Yushi, Fukuroi, Japan).

\section{Statistical analysis}

Results are presented as mean \pm s.d. Unpaired Student's $t$-test was used for comparison of mean values of parametric continuous variables between smoker and control groups. Because of asymmetric distribution, values for ROCK activity and the protein expression of MBS, ROCK1 and ROCK2 were analyzed using nonparametric methods. For nonparametric analysis, we used MannWhitney $U$-test to evaluate the difference of levels between groups. All reported $P$-values were two sided, and a $P$-value of $<0.05$ was considered statistically significant. Multivariate analysis was performed with the Statistical Analysis System program package (SAS Institute, Cary, NC, USA). The data were analyzed using the software package StatView V (SAS Institute) and Super ANOVA (Abacus Concepts, Berkley, CA, USA).

\section{RESULTS}

\section{Clinical characteristics}

Baseline clinical characteristics of the 14 smokers and 15 non-smokers are summarized in the Table 1. Serum concentration of 8-OHdG was significantly higher in smokers than in non-smokers. There was no significant difference in other parameters between the smokers and non-smokers.

\section{ROCK activity}

ROCK activity in peripheral blood leukocytes was significantly higher in smokers than in non-smokers $(0.78 \pm 0.27$ vs. $0.54 \pm 018, P=0.012$; Figure $1 \mathrm{~b}$ ). Protein expression of MBS, ROCK1 and ROCK2 were

Table 1 Clinical characteristics in non-smokers and smokers

\begin{tabular}{|c|c|c|}
\hline Variables & $\begin{array}{l}\text { Non-smoker } \\
(\mathrm{n}=15)\end{array}$ & $\begin{array}{l}\text { Smoker } \\
(\mathrm{n}=14)\end{array}$ \\
\hline Age (years) & $34.8 \pm 6.5$ & $35.8 \pm 3.8$ \\
\hline Body mass index $\left(\mathrm{kg} \mathrm{cm}^{-2}\right)$ & $22.3 \pm 1.7$ & $23.7 \pm 2.8$ \\
\hline Systolic blood pressure (mm Hg) & $120.1 \pm 14.4$ & $117.6 \pm 11.9$ \\
\hline Diastolic blood pressure (mm Hg) & $65.7 \pm 9.4$ & $67.5 \pm 10.5$ \\
\hline Heart rate (beats $\mathrm{min}^{-1}$ ) & $65.8 \pm 9.6$ & $78.3 \pm 7.6$ \\
\hline Total cholesterol (mg per $100 \mathrm{ml}$ ) & $184.1 \pm 32.7$ & $206.4 \pm 31.1$ \\
\hline Triglycerides (mg per $100 \mathrm{ml}$ ) & $126.9 \pm 96.6$ & $144.4 \pm 66.2$ \\
\hline $\begin{array}{l}\text { Low-density lipoprotein cholesterol (mg per } \\
100 \mathrm{ml} \text { ) }\end{array}$ & $112.4 \pm 30.4$ & $113 \pm 30.4$ \\
\hline $\begin{array}{l}\text { High-density lipoprotein cholesterol (mg per } \\
100 \mathrm{ml} \text { ) }\end{array}$ & $57.9 \pm 13.7$ & $61.8 \pm 22.2$ \\
\hline Fasting serum glucose (mg per $100 \mathrm{ml}$ ) & $98.9 \pm 14.9$ & $108.6 \pm 10.3$ \\
\hline Serum creatinine (mg per $100 \mathrm{ml}$ ) & $0.80 \pm 0.09$ & $0.84 \pm 0.06$ \\
\hline $\begin{array}{l}\text { High-sensitivity C-reactive protein (mg per } \\
100 \mathrm{ml} \text { ) }\end{array}$ & $0.05 \pm 0.08$ & $0.06 \pm 0.05$ \\
\hline 8-hydroxy-2'-deoxyguanosine (ng ml-1) & $0.22 \pm 0.03$ & $0.28 \pm 0.04 *$ \\
\hline
\end{tabular}

similar for the smokers and non-smokers $(1.91 \pm 0.87$ vs. $2.04 \pm 1.60$, $1.54 \pm 0.85$ vs. $1.95 \pm 1.12$, and $1.45 \pm 0.56$ vs. $1.29 \pm 0.14$, respectively, Figures $1 \mathrm{c}$ and $\mathrm{d}$ ).

\section{Relationship between ROCK activity and parameters}

Using univariate linear regression analysis, only systolic blood pressure significantly correlated with ROCK activity $(r=0.42, P=0.039$; Table 2 and Figure 2). Multivariate analysis was performed to explain variability (systolic blood pressure and smoking status being associated with ROCK activity) of ROCK activity. Smoking status was an independent predictor of ROCK activity (Table 3 ).

\section{Vascular function}

FMD was significantly lower in smokers than in non-smokers ( $4.7 \pm 3.1$ vs. $9.0 \pm 3.8 \%, P=0.005$; Figure $3 \mathrm{a}$ ). NTG-induced vasodilation was similar in smokers and non-smokers $(16.5 \pm 5.8 \mathrm{vs}$. $13.7 \pm 5.1 \%$; Figure $3 \mathrm{~b}$ ). Baseline arterial diameter and peak hyperemic blood flow were similar in the two groups $(4.3 \pm 0.5 \mathrm{vs} .4 .0 \pm 0.4 \mathrm{~mm}$ and $306 \pm 149$ vs. $362 \pm 167 \%$, respectively).

\section{Relationship between ROCK activity and FMD}

There was a significant correlation between ROCK activity and FMD $(r=-0.42 ; P=0.035$; Figure 4$)$, whereas ROCK activity did not correlate with NTG-induced vasodilation. Using univariate linear regression analysis, no variable other than ROCK activity significantly correlated with FMD (Table 4). There was no significant relationship between NTG-induced vasodilation and parameters.

\section{DISCUSSION}

In this present study we showed that ROCK activity in peripheral blood leukocytes was higher in young male smokers than in nonsmokers and was inversely correlated with FMD. Multiple regression analysis revealed that smoking status was an independent predictor of ROCK activity.

We selected young men to avoid the possibility of alteration in ROCK activity and endothelial function caused by factors other than smoking, such as hypertension, heart failure, atherosclerosis, hypercholesterolemia, diabetes mellitus, aging and menstrual cycle.

We have recently reported that intra-arterial infusion of fasudil, which is a broad ROCK inhibitor, increases forearm blood flow in smokers but not in non-smokers, suggesting that ROCK activity may be higher in smokers than in non-smokers. ${ }^{22}$ In this study, we confirmed that ROCK activity was increased in smokers using a noninvasive method for measurement of ROCK activity in peripheral blood leukocytes. It is well known that peripheral blood leukocytes per se have an important role in the cause, maintenance and development of atherosclerosis. Therefore, our findings suggest that the enhancement of ROCK activity by cigarette smoking may cause leukocyte activation and enhanced leukocyte infiltration into the vascular wall, leading to progression of atherosclerosis. Furthermore, leukocyte ROCK activity has been shown to correlate with endothelial dysfunction. ${ }^{11}$ It is clinically important to estimate the degree of ROCK activity. A noninvasive method for measuring leukocyte ROCK activity would also be useful for assessing ROCK activity. Leukocyte ROCK activity may become a novel biomarker of endothelial function and atherosclerosis.

Inflammation has a critical role in the development and complications of atherosclerosis. ${ }^{13,23}$ Previous studies have shown that cigarette smoking causes inflammation and increases the numbers of circulating leukocytes and circulating inflammatory markers such as CRP, interleukin-6 and soluble intercellular adhesion molecule type $1 .^{6}$ 
a

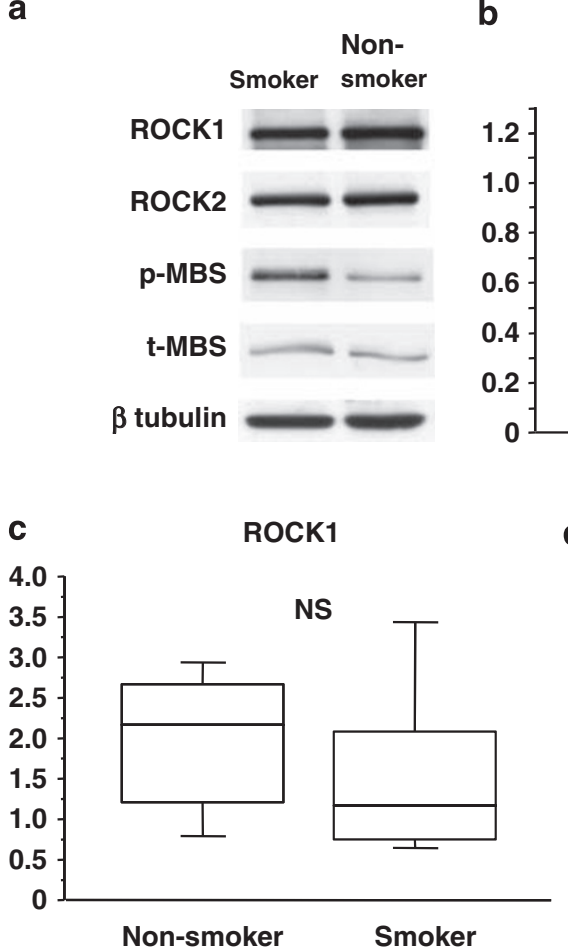

ROCK activity

(p-MBS/t-MBS)

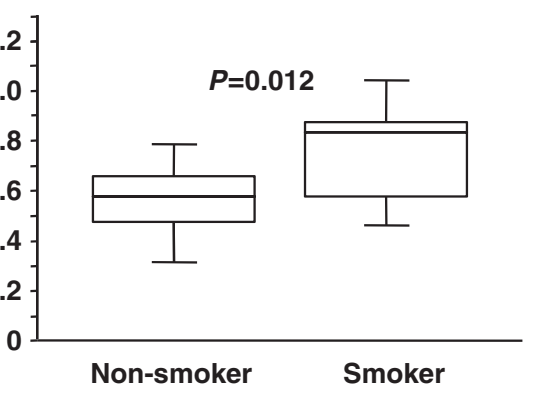

d

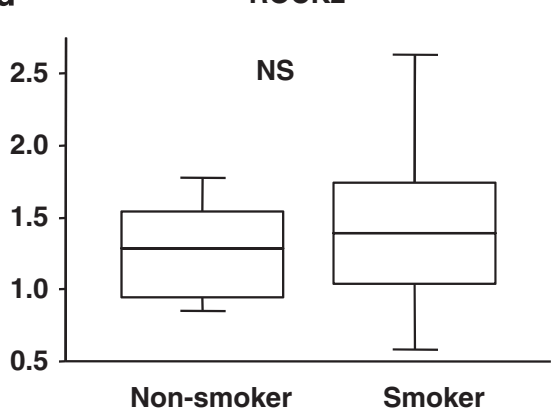

Figure 1 Rho-associated kinase (ROCK) activity in smokers and non-smokers. (a) Western blot analysis for ROCK1, ROCK2, phospho-myosin-binding subunit (p-MBS), total-myosin-binding subunit (t-MBS) and $\beta$-tubulin. (b) ROCK activity (p-MBS/t-MBS) in smokers and non-smokers. (c) ROCK1 expression in smokers and non-smokers. (d) ROCK2 expression in smokers and non-smokers.

Table 2 Simple regression analysis between ROCK activity and variables

\begin{tabular}{|c|c|c|}
\hline Variables & Coefficient & P-value \\
\hline Age (years) & 0.04 & 0.86 \\
\hline Body mass index $\left(\mathrm{kg} \mathrm{cm}^{-2}\right)$ & 0.32 & 0.11 \\
\hline Systolic blood pressure (mm Hg) & 0.42 & 0.04 \\
\hline Diastolic blood pressure (mm Hg) & 0.08 & 0.94 \\
\hline Heart rate (beats $\mathrm{min}^{-1}$ ) & 0.06 & 0.80 \\
\hline Total cholesterol (mg per $100 \mathrm{ml}$ ) & 0.35 & 0.10 \\
\hline Triglycerides (mg per $100 \mathrm{ml}$ ) & 0.19 & 0.39 \\
\hline Low-density lipoprotein cholesterol (mg per 100 ml) & 0.28 & 0.18 \\
\hline High-density lipoprotein cholesterol (mg per 100 ml) & 0.03 & 0.80 \\
\hline Fasting serum glucose (mg per $100 \mathrm{ml}$ ) & 0.24 & 0.23 \\
\hline Serum creatinine (mg per 100 ml) & 0.11 & 0.62 \\
\hline High-sensitivity C-reactive protein (mg per 100 ml) & 0.10 & 0.64 \\
\hline 8-hydroxy-2'-deoxyguanosine (ng ml-1) & 0.36 & 0.20 \\
\hline
\end{tabular}

Abbreviation: ROCK, Rho-associated kinase.

Activation of circulating leukocytes leading to the recruitment and infiltration of inflammatory cells into vessel walls has an important role in the pathogenesis of atherosclerosis. ${ }^{4,24}$ ROCKs regulate inflammatory cell accumulation in the vessel wall and contribute to the development of vascular inflammation and remodeling. ${ }^{13,25}$ Although the mechanism by which smoking enhances ROCK activity in leukocytes remains unclear, smoking may increase leukocytes chemotaxis and recruitment to the vessel wall through activation of ROCKs. Indeed, deletion of ROCK1 leads to impaired leukocyte chemotaxis and migration. ${ }^{25,26}$ In this study, there was no significant difference in high-sensitivity CRP between the smokers and non-smokers.

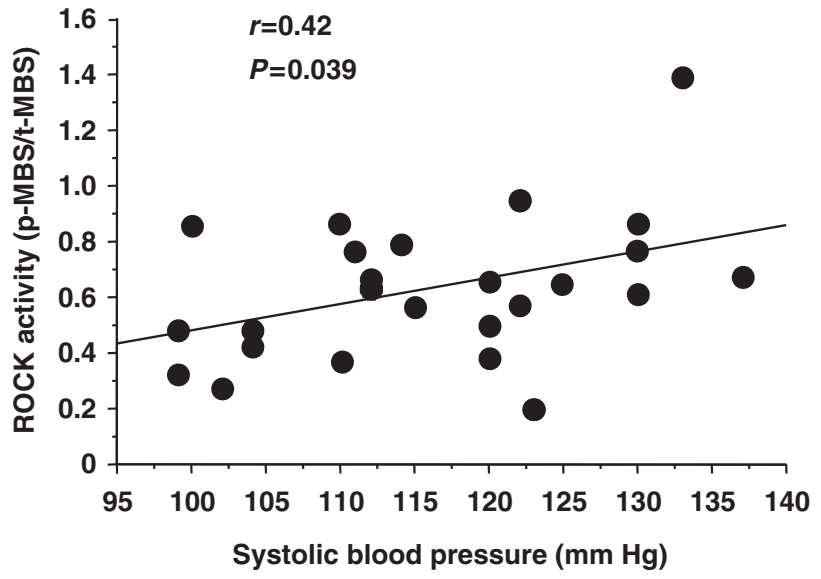

Figure 2 Relationship between Rho-associated kinase (ROCK) activity and systolic blood pressure.

In addition, there was no significant relationship between the number of cigarettes (pack-year) and CRP levels.

Several investigators have shown an interaction between the RhoA/ ROCk pathway and reactive oxygen species. ${ }^{27}$ Indeed, reactive oxygen species induced by hyperglycemia enhances ROCK activity, leading to atherothrombogenesis through an increase in expression of plasminogen activator inhibitor-1 in vascular endothelial cells. ${ }^{28}$ It is well known that cigarette smoking decreases NO bioavailability through the production of reactive oxygen species. Several investigators, including us, have shown that there is a possible association of ROCK activity with oxidative stress and that smoking enhances the 
Table 3 Multiple regression analysis for ROCK activity

\begin{tabular}{lcccc}
\hline Variables & Coefficient & s.e. & \multicolumn{1}{c}{$95 \% \mathrm{Cl}$} & P-value \\
\hline Intercept & -0.34 & 0.45 & $-1.27-0.59$ & 0.46 \\
Systolic blood pressure & 0.01 & 0.003 & $-0.001-0.026$ & 0.06 \\
Smoking & 0.22 & 0.08 & $0.05-0.38$ & 0.01 \\
\hline
\end{tabular}

Abbreviations: $\mathrm{Cl}$, confidence interval; ROCK, Rho-associated kinase.

a
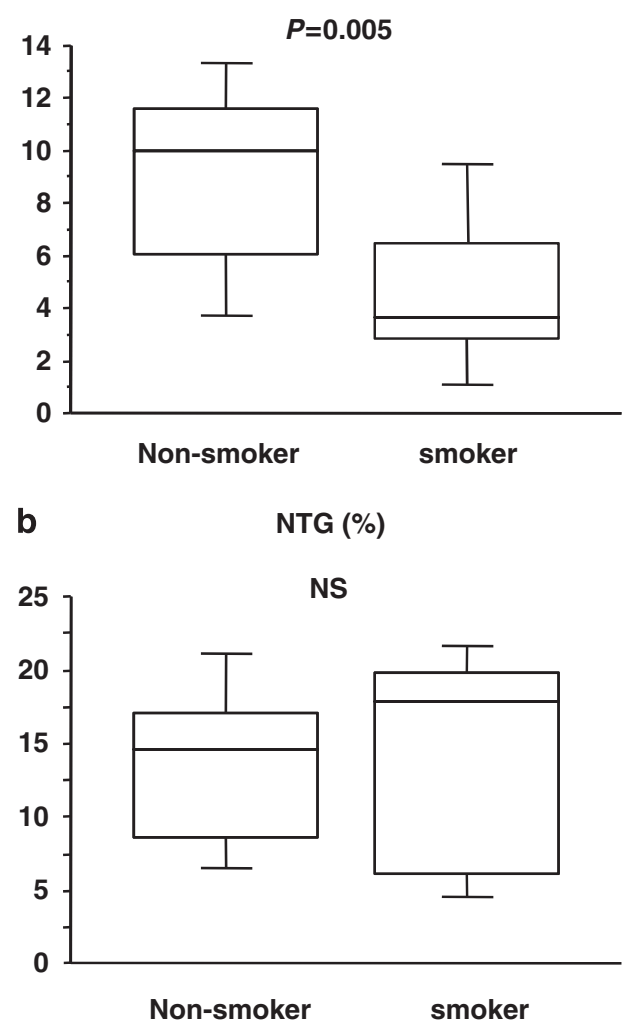

Figure 3 Vascular function in smokers and non-smokers. (a) Flow-mediated vasodilation (FMD) in the smokers and non-smokers. (b) Nitroglycerine (NTG)-induced vasodilation in smokers and non-smokers.

activation of ROCKs in vascular smooth muscle cells in vivo and in vitro. ${ }^{22,27,29}$ Excess oxidative stress may have a role in the smokinginduced activation of ROCKs in leukocytes. Increase in ROCK activity in vascular endothelial cells directly alters FMD through the inactivation of eNOS. It is well known that activation of ROCK induces various substrates and transcriptional factors, leading to actin cytoskeleton organization, smooth muscle contraction, vascular inflammation, apoptosis and gene expression differentiation. Under the condition of excessive reactive oxygen species by smoking, oxidative stress and activated ROCK-induced impairment of various cellular functions may cause a vicious circle. Thus, activated ROCK in vascular smooth muscle cells and in leukocytes may also contribute to impaired FMD through increase in oxidative stress. In this study, serum concentration of 8-OHdG was significantly higher in smokers than in non-smokers. However, univariate and multiple regression analyses revealed that 8 -OHdG was not an independent predictor of ROCK activity.

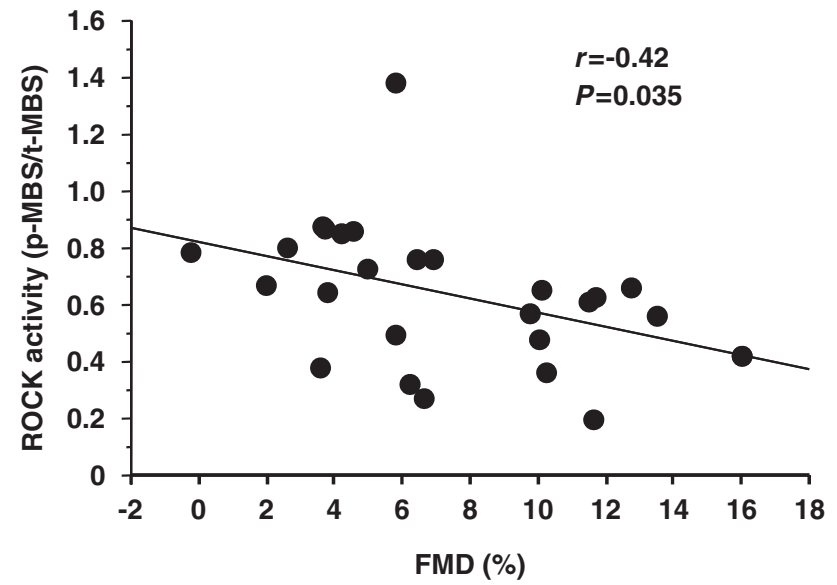

Figure 4 Relationship between Rho-associated kinase (ROCK) activity and flow-mediated vasodilation (FMD).

Table 4 Simple regression analysis between FMD activity and variables

\begin{tabular}{|c|c|c|}
\hline Variables & Coefficient & P-value \\
\hline Age (years) & 0.12 & 0.56 \\
\hline Body mass index $\left(\mathrm{kg} \mathrm{cm}^{-2}\right)$ & 0.17 & 0.44 \\
\hline Systolic blood pressure (mm Hg) & 0.22 & 0.30 \\
\hline Diastolic blood pressure (mm Hg) & 0.17 & 0.36 \\
\hline Heart rate (beats $\mathrm{min}^{-1}$ ) & 0.32 & 0.16 \\
\hline Total cholesterol (mg per $100 \mathrm{ml}$ ) & 0.39 & 0.09 \\
\hline Triglycerides (mg per $100 \mathrm{ml}$ ) & 0.23 & 0.32 \\
\hline Low-density lipoprotein cholesterol (mg per 100 ml) & 0.23 & 0.33 \\
\hline High-density lipoprotein cholesterol (mg per $100 \mathrm{ml}$ ) & 0.36 & 0.12 \\
\hline Fasting serum glucose (mg per $100 \mathrm{ml})$ & 0.16 & 0.47 \\
\hline Serum creatinine (mg per $100 \mathrm{ml}$ ) & 0.17 & 0.48 \\
\hline High-sensitivity C-reactive protein (mg per 100 ml) & 0.18 & 0.45 \\
\hline 8-hydroxy-2'-deoxyguanosine (ng ml-1) & 0.16 & 0.63 \\
\hline Rho-associated kinase activity & 0.42 & 0.03 \\
\hline
\end{tabular}

Abbreviation: FMD, flow-mediated vasodilation.

Interestingly, ROCK activity significantly correlated with systolic blood pressure even in the young male subjects with normal blood pressure. This finding is supported by results of previous studies showing that the ROCKs are involved in the pathogenesis of hypertension through, at least in part, causing vascular smooth muscle cell contraction..$^{9,16,30}$ There is a possibility that ROCK activity regulates blood pressure from the normal range to high levels. However, multivariate regression analysis revealed that smoking but not blood pressures independently correlated with ROCK activity.

FMD, as an index of endothelium-dependent vasodilation, was impaired even in young smokers compared with non-smokers. These findings are consistent with results of previous studies showing that smoking is associated with endothelial dysfunction.,22 One of the important findings in this study is a significant relationship between ROCK activity in peripheral leukocytes and FMD in smokers. As Sauzeau et al. ${ }^{30}$ have shown in vascular smooth muscle cells, NO may also inhibit RhoA translocation from the cytosol to membrane in leukocytes. In addition, previous studies have shown that activation of the RhoA/ROCK pathway impairs NO bioavailability through the inhibition of eNOS mRNA stability, eNOS posphorylation at Ser 1177, Akt/phosphoinositide 3-kinase pathway and enhancement of eNOS 
phosphorylation at Thr495. ${ }^{20,22}$ Taken together, the results indicate that ROCK activity in leukocytes may reflect ROCK activity in vascular endothelial cells and endothelial function, suggesting an interaction between ROCK activity and endogenous NO.

\section{Limitation}

We hypothesized that smoking causes inflammation, leading to enhancement of ROCK activity. However, serum levels of CRP, which is a marker of systemic inflammation, were similar in smokers and non-smokers. The discrepancy in the effect of smoking on inflammation between the present study and previous studies may be due to the limited number of subjects in each group, differences in age (our subjects being younger), the magnitude of smoking (our subjects having a smaller number of pack-years) and body mass index BMI (our subjects having smaller body mass index). ${ }^{9,21}$ In addition, the levels of CRP in smokers and non-smokers in this study were $<0.2 \mathrm{mg}$ per $100 \mathrm{ml}$. The levels of CRP in this study were lower than those in previous studies showing a relationship between smoking and CRP levels. ${ }^{9,21}$ In those previous studies, the levels of CRP in smokers were $>0.2 \mathrm{mg}$ per $100 \mathrm{ml}$. However, we cannot exclude the possibility that inflammation caused by smoking contributes to the enhancement of ROCK activity. Further studies are required with other inflammatory biomarkers to determine whether there is any relationship between inflammation and ROCK activity.

In conclusion, measurement of ROCK activity in peripheral leukocytes is minimally invasive and does not require pharmacologic intervention (for example, intra-arterial or intravenous infusion of ROCK inhibitors) and may be useful as a novel predictor of atherosclerotic disease in a clinical setting. It remains to be determined whether ROCK activity is a predictor of cardiovascular outcome.

\section{CONFLICT OF INTEREST}

Dr James K Liao is a consultant for Asahi-Kasei Pharmaceutical.

\section{ACKNOWLEDGEMENTS}

We thank Megumi Wakisaka, Keiko Umeda and Satoko Michiyama for their excellent secretarial assistance. This study was supported in part by a Grant-inAid for Scientific Research from the Ministry of Education, Science and Culture of Japan (1559075100 and 1859081500), and the National Institutes of Health (HL052233 and HL08187).

1 Howard G, Wagenknecht LE, Burke GL, Diez-Roux A, Evans GW, McGovern P, Nieto FJ, Tell GS. Cigarette smoking and progression of atherosclerosis: the Atherosclerosis Risk in Communities (ARIC) study. JAMA 1998; 279: 119-124.

2 Tell GS, Polak JF, Ward BJ, Kittner SJ, Savage PJ, Robbins J. Relation of smoking with carotid artery wall thickness and stenosis in older adults. The Cardiovascular Health Study. The Cardiovascular Health Study (CHS) Collaborative Research Group. Circulation 1994; 90: 2905-2908.

3 Barua RS, Ambrose JA, Eales-Reynolds LJ, DeVoe MC, Zervas JG, Saha DC. Dysfunctional endothelial nitric oxide biosynthesis in healthy smokers with impaired endothelium-dependent vasodilatation. Circulation 2001; 104: 1905-1910.

4 Libby P, Ridker PM, Maseri A. Inflammation and atherosclerosis. Circulation 2002; 105: 1135-1143.

5 Celermajer DS, Adams MR, Clarkson P, Robinson J, McCredie R, Donald A, Deanfield JE. Passive smoking and impaired endothelium-dependent arterial dilatation in healthy young adults. N Engl J Med 1996; 334: 150-155.

6 Tracy RP, Psaty BM, Macy E, Bovill EG, Cushman M, Cornell ES, Kuller LH. Lifetime smoking exposure affects the association of C-reactive protein with cardiovascular disease risk factors and subclinical disease in healthy elderly subjects. Arterioscler Thromb Vasc Biol 1997; 17: 2167-2176.

7 Bermudez EA, Rifai N, Buring JE, Manson JE, Ridker PM. Relation between markers of systemic vascular inflammation and smoking in women. Am J Cardiol 2002; 89: 1117-1119

8 Amano M, Chihara K, Kimura K, Fukata Y, Nakamura N, Matsuura Y, Kaibuchi K. Formation of actin stress fibers and focal adhesions enhanced by Rho-kinase. Science 1997; 275: 1308-1311.

9 Uehata M, Ishizaki T, Satoh H, Ono T, Kawahara T, Morishita T, Tamakawa H, Yamagami K, Inui J, Maekawa M, Narumiya S. Calcium sensitization of smooth muscle mediated by a Rho-associated protein kinase in hypertension. Nature 1997; 389: 990-994.

10 Hall A. Rho GTPases and the actin cytoskeleton. Science 1998; 279: 509-514.

11 Nohria A, Grunert ME, Rikitake Y, Noma K, Prsic A, Ganz P, Liao JK, Creager MA. Rho kinase inhibition improves endothelial function in human subjects with coronary artery disease. Circ Res 2006; 99: 1426-1432.

12 Kimura K, Fukata Y, Matsuoka Y, Bennett V, Matsuura Y, Okawa K, Iwamatsu A, Kaibuchi K. Regulation of the association of adducin with actin filaments by Rhoassociated kinase (Rho-kinase) and myosin phosphatase. J Biol Chem 1998; 273: 5542-5548.

13 Mallat Z, Gojova A, Sauzeau V, Brun V, Silvestre JS, Esposito B, Merval R, Groux H, Loirand G, Tedgui A. Rho-associated protein kinase contributes to early atherosclerotic lesion formation in mice. Circ Res 2003; 93: 884-888.

14 Seasholtz TM, Wessel J, Rao F, Rana BK, Khandrika S, Kennedy BP, Lillie EO, Ziegler MG, Smith DW, Schork NJ, Brown JH, O'Connor DT. Rho kinase polymorphism influences blood pressure and systemic vascular resistance in human twins: role of heredity. Hypertension 2006; 47: 937-947.

15 Rikitake Y, Oyama N, Wang CY, Noma K, Satoh M, Kim HH, Liao JK. Decreased perivascular fibrosis but not cardiac hypertrophy in ROCK1+/- haploinsufficient mice. Circulation 2005; 112: 2959-2965.

16 Masumoto A, Hirooka Y, Shimokawa H, Hironaga K, Setoguchi S, Takeshita A. Possible involvement of Rho-kinase in the pathogenesis of hypertension in humans. Hypertension 2001; 38: 1307-1310.

17 Masumoto A, Mohri M, Shimokawa H, Urakami L, Usui M, Takeshita A. Suppression of coronary artery spasm by the Rho-kinase inhibitor fasudil in patients with vasospastic angina. Circulation 2002; 105: 1545-1547.

18 Vane JR, Anggard EE, Botting RM. Regulatory functions of the vascular endothelium. $N$ Engl J Med 1990; 323: 27-36.

19 Vanhoutte PM. Endothelium and control of vascular function. State of the art lecture. Hypertension 1989; 13(6 Pt 2): 658-667.

20 Wolfrum S, Dendorfer A, Rikitake Y, Stalker TJ, Gong Y, Scalia R, Dominiak P, Liao JK Inhibition of Rho-kinase leads to rapid activation of phosphatidylinositol 3-kinase/ protein kinase Akt and cardiovascular protection. Arterioscler Thromb Vasc Biol 2004; 24: 1842-1847.

21 Liu PY, Chen JH, Lin LJ, Liao JK Increased Rho kinase activity in a Taiwanese population with metabolic syndrome. J Am Coll Cardiol 2007; 49: 1619-1624.

22 Noma K, Higashi Y, Jitsuiki D, Hara K, Kimura M, Nakagawa K, Goto C, Oshima T, Yoshizumi M, Chayama K. Smoking activates rho-kinase in smooth muscle cells of forearm vasculature in humans. Hypertension 2003; 41: 1102-1105.

23 Lusis AJ. Atherosclerosis. Nature 2000; 407: 233-241.

24 Serrano Jr CV, Ramires JA, Venturinelli M, Arie S, D’Amico E, Zweier JL, Pileggi F, da Luz PL. Coronary angioplasty results in leukocyte and platelet activation with adhesion molecule expression. Evidence of inflammatory responses in coronary angioplasty. J Am Coll Cardiol 1997; 29: 1276-1283.

25 Noma K, Rikitake Y, Oyama N, Yan G, Alcaide P, Liu PY, Wang H, Ahl D, Sawada N, Okamoto R, Hiroi Y, Shimizu K, Luscinskas FW, Sun J, Liao JK. ROCK1 mediates leukocyte recruitment and neointima formation following vascular injury. J Clin Invest 2008.

26 Wang HW, Liu PY, Oyama N, Rikitake Y, Kitamoto S, Gitlin J, Liao JK, Boisvert WA. Deficiency of ROCK1 in bone marrow-derived cells protects against atherosclerosis in LDLR-/- mice. FASEB J 2008; 22: 3561-3570.

27 Bailey SR, Mitra S, Flavahan S, Flavahan NA. Reactive oxygen species from smooth muscle mitochondria initiate cold-induced constriction of cutaneous arteries. $A m \mathrm{~J}$ Physiol Heart Circ Physiol 2005; 289: H243-H250.

28 Rikitake Y, Liao JK. Rho-kinase mediates hyperglycemia-induced plasminogen activator inhibitor-1 expression in vascular endothelial cells. Circulation 2005; 111 : 3261-3268.

29 Noma K, Goto C, Nishioka K, Jitsuiki D, Umemura T, Ueda K, Kimura M, Nakagawa K, Oshima T, Chayama K, Yoshizumi M, Liao JK, Higashi Y. Roles of rho-associated kinase and oxidative stress in the pathogenesis of aortic stiffness. J Am Coll Cardiol 2007; 49. 698-705.

30 Sauzeau V, Le Jeune H, Cario-Toumaniantz C, Smolenski A, Lohmann SM, Bertoglio J, Chardin P, Pacaud P, Loirand G. Cyclic GMP-dependent protein kinase signaling pathway inhibits RhoA-induced $\mathrm{Ca} 2+$ sensitization of contraction in vascular smooth muscle. J Biol Chem 2000; 275: 21722-21729. 